\title{
Friomaramide, a highly modified linear hexapeptide from an Antarctic sponge, inhibits Plasmodium
} falciparum liver stage development.

Matthew A. Knestrick, ${ }^{\dagger}$ Nerida G. Wilson, ${ }^{\ddagger}$ Alison Roth, ${ }^{\S}$ John H. Adams, ${ }^{\S}$ and Bill J. Baker*†

${ }^{\dagger}$ Department of Chemistry, University of South Florida, 4202 E. Fowler Ave., CHE205, Tampa, Florida 33620, United States

${ }^{\ddagger}$ Western Australia Museum and University of Western Australia Perth, Western Australia, Australia

${ }^{\S}$ Center for Global Health \& Infectious Diseases Research, Department of Global Health, College of Public Health, University of South Florida, Tampa, Florida, United States.

Figure S1. ${ }^{1} \mathrm{H}$ NMR spectrum of friomaramide in $\mathrm{MeOH}-d_{4}, 800 \mathrm{MHz}$....................................2

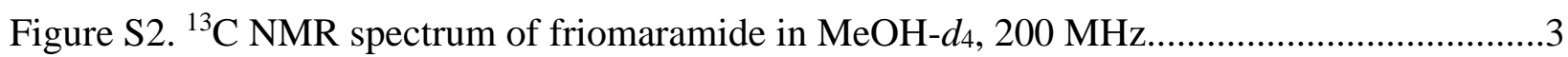

Figure S3. gHSQC NMR spectrum of friomaramide in $\mathrm{MeOH}-d_{4}, 800 \mathrm{MHz}$............................4

Figure S4. gCOSY NMR spectrum of friomaramide in $\mathrm{MeOH}-d_{4}, 800 \mathrm{MHz}$............................5

Figure S5. gHMBC NMR spectrum of friomaramide in $\mathrm{MeOH}-d_{4}, 800 \mathrm{MHz}$...........................6

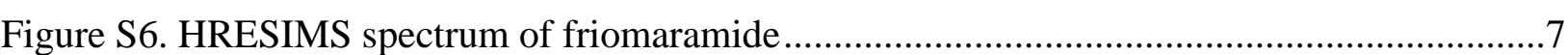

Table S1. Samples used in this study (accessioned at Scripps Institution of Oceanography, Benthic Invertebrate Collection).. .8

Table S2. Marfey’s analysis of friomaramide. . .8 


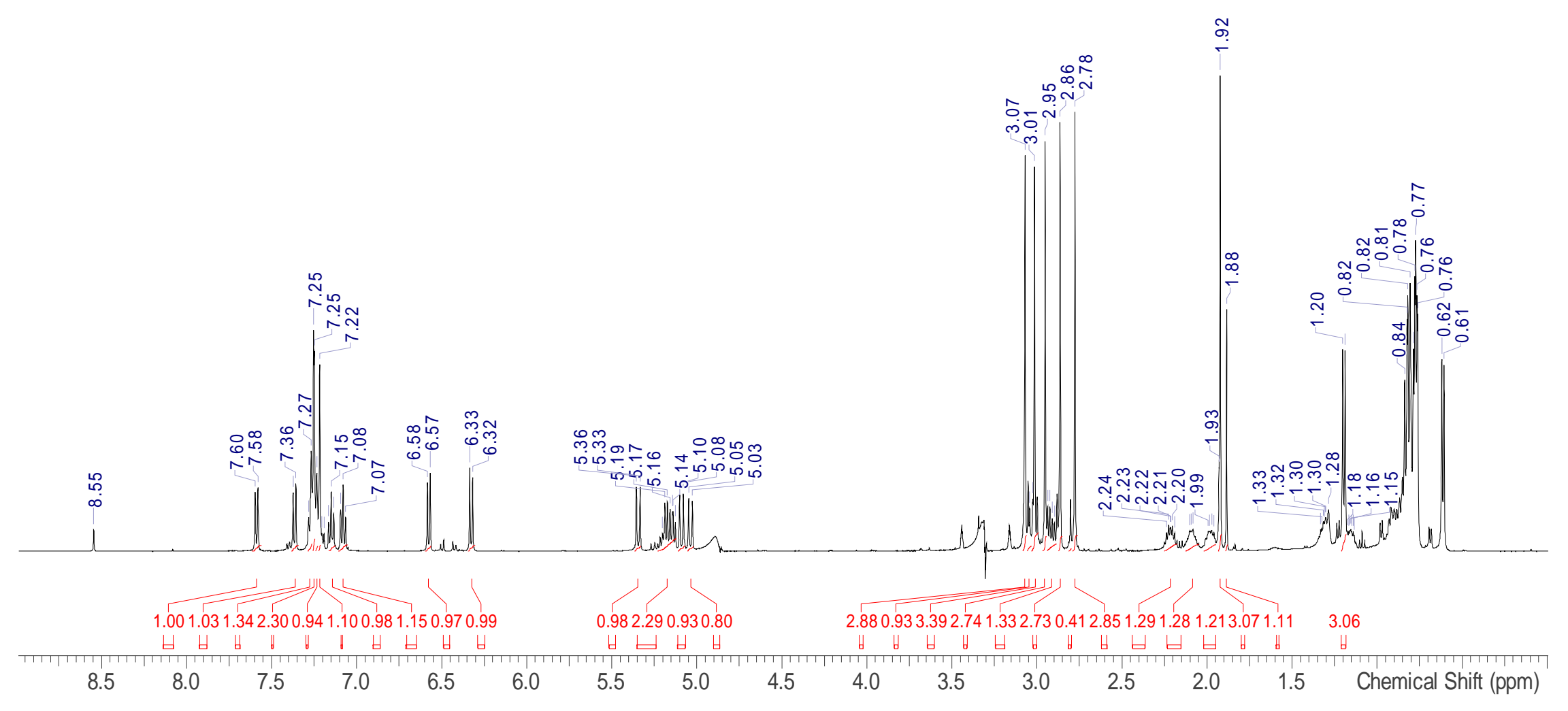

Figure S1. ${ }^{1} \mathrm{H}$ NMR spectrum of friomaramide in $\mathrm{MeOH}-d_{4}, 800 \mathrm{MHz}$. 


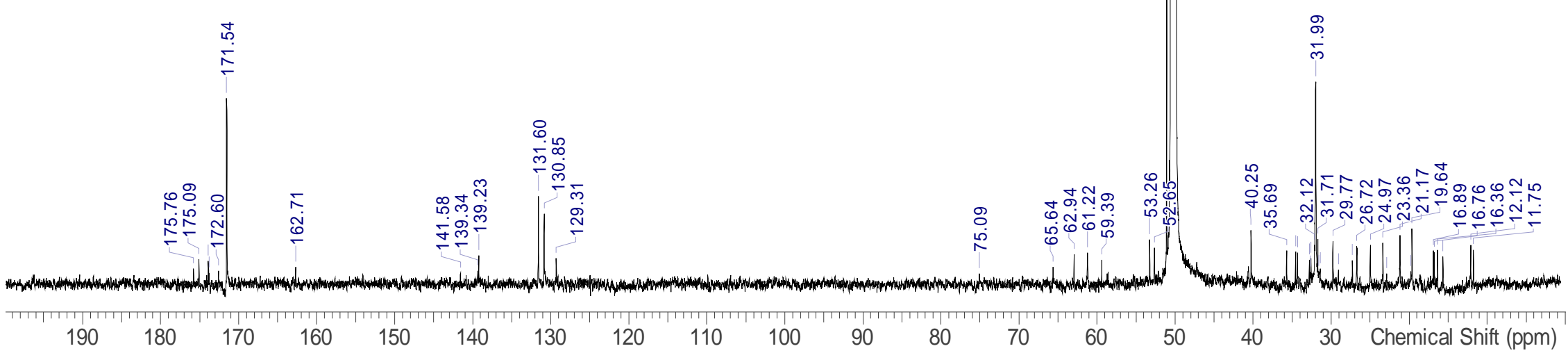

Figure S2. ${ }^{13} \mathrm{C}$ NMR spectrum of friomaramide in $\mathrm{MeOH}-d_{4}, 200 \mathrm{MHz}$. 


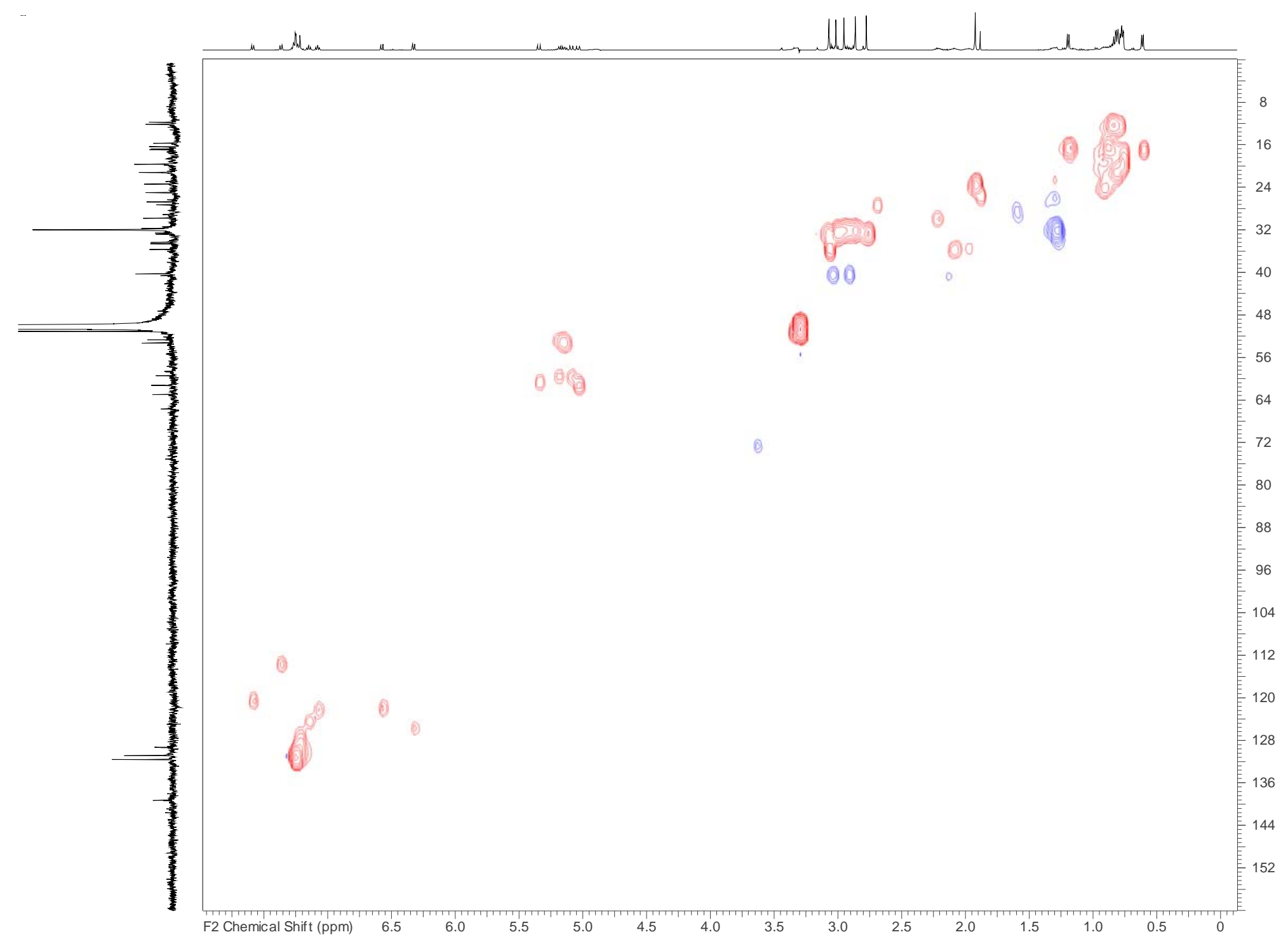

Figure S3. gHSQC NMR spectrum of friomaramide in $\mathrm{MeOH}-d_{4}, 800 \mathrm{MHz}$. 


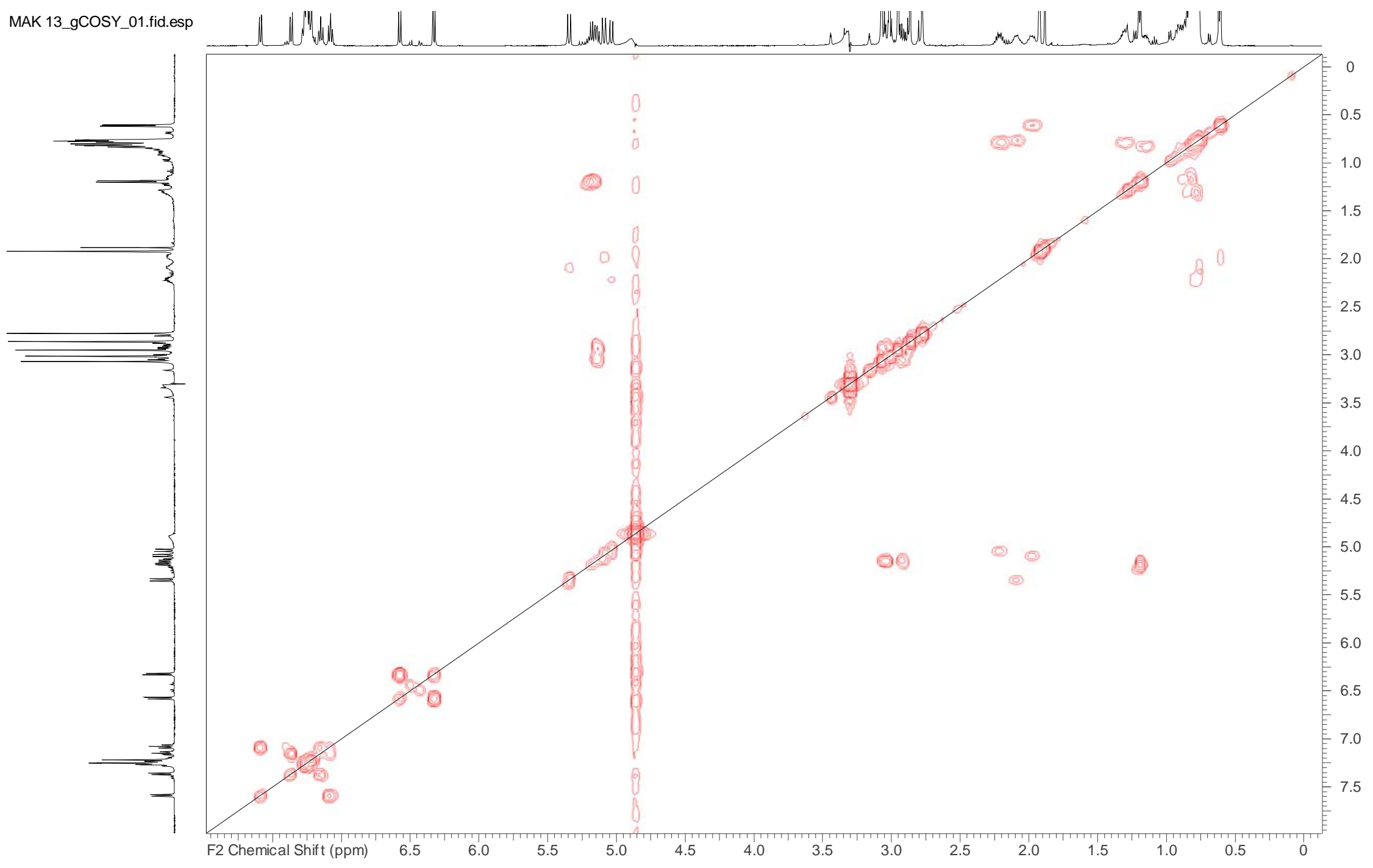

Figure S4. gCOSY NMR spectrum of friomaramide in $\mathrm{MeOH}-d_{4}, 800 \mathrm{MHz}$. 


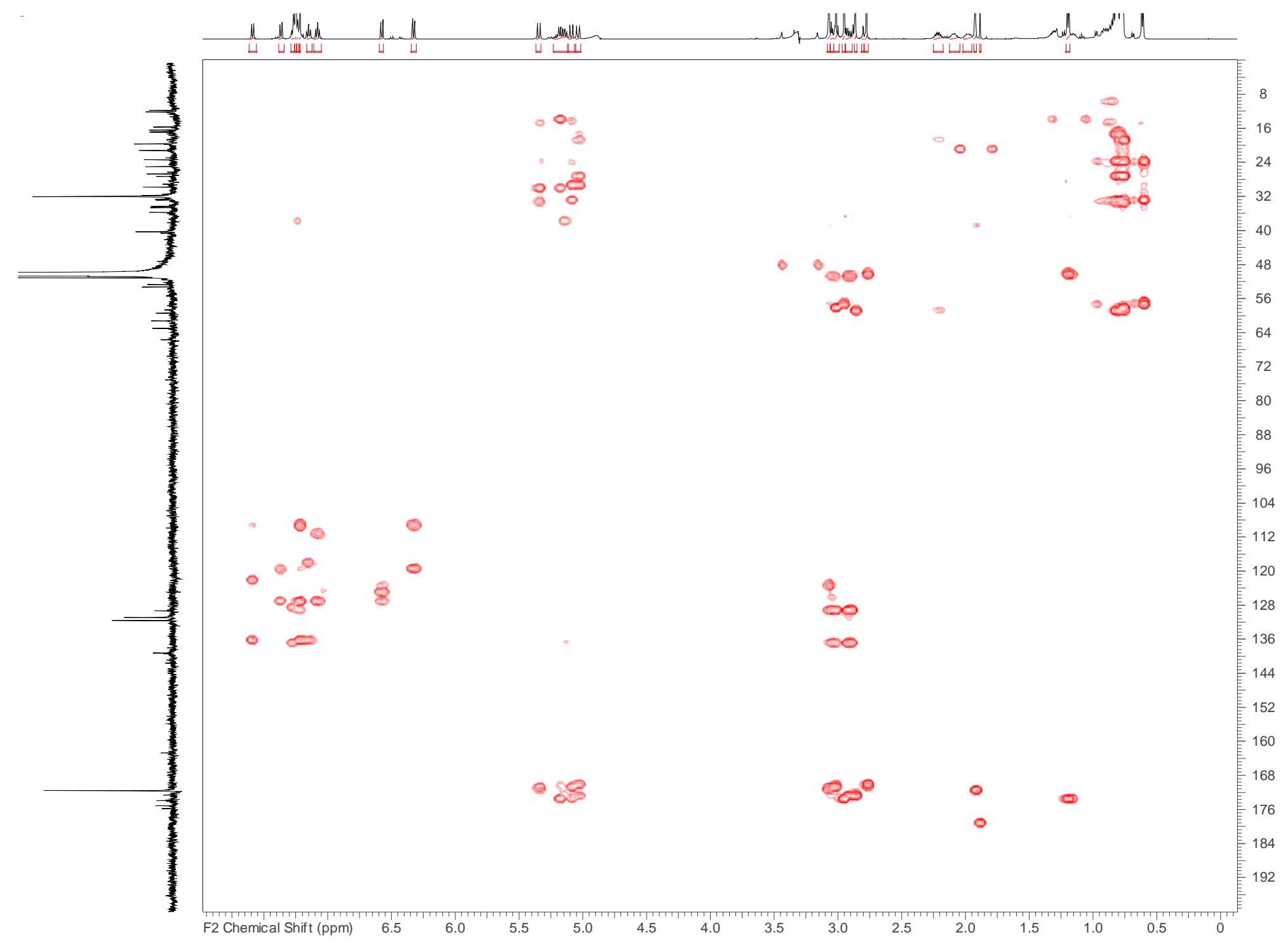

Figure S5. gHMBC NMR spectrum of friomaramide in MeOH- $d_{4}, 800 \mathrm{MHz}$. 
MFE MS Spectrum

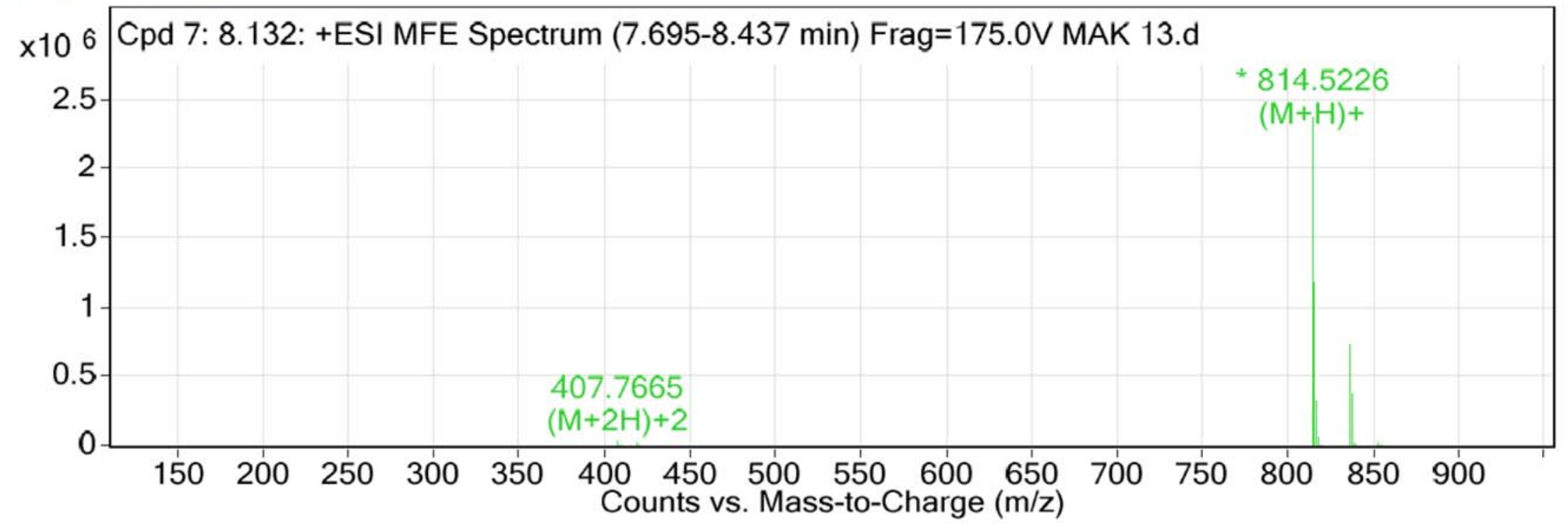

Figure S6. HRESIMS spectrum of friomaramide. 
Table S1. Samples used in this study (accessioned at Scripps Institution of Oceanography, Benthic Invertebrate Collection) for the isolation of friomaramide ("cold water amide”).

\begin{tabular}{|l|l|l|}
\hline Field number & Location & Depth $(\mathrm{m})$ \\
\hline S20232 & South Georgia & 394 \\
\hline S20375 & Shag Rocks & 127 \\
\hline S20399 & South Georgia & 137 \\
\hline S20400 & South Georgia & 137 \\
\hline S20411 & Shag Rocks & 129 \\
\hline S20437 & Shag Rocks & 127 \\
\hline S20454 & Shag Rocks & 130 \\
\hline
\end{tabular}
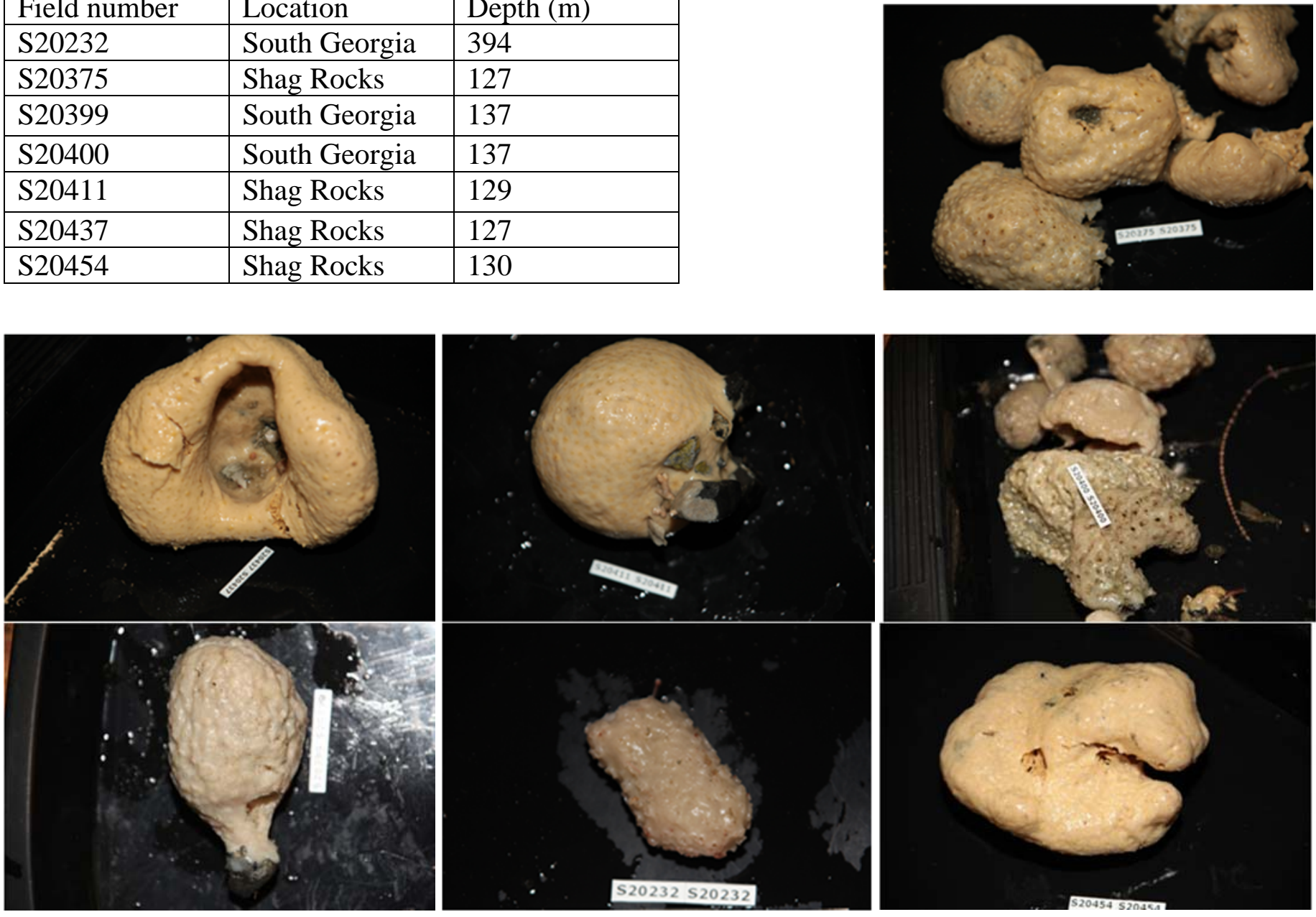
Table S2. Marfey’s analysis of friomaramide.

\begin{tabular}{lll}
\hline $\begin{array}{l}\boldsymbol{N} \text {-Me } \\
\text { Amino Acid }\end{array}$ & $\begin{array}{l}\text { Retention } \\
\text { Time (min) }\end{array}$ & $\begin{array}{l}\text { Friomaramide Amino } \\
\text { Acid Retention Time (min) }\end{array}$ \\
\hline D-Ala & 5.77 & \\
L-Ala & 5.91 & 5.89 \\
D-Ile & 6.94 & \\
L-Ile & 7.57 & 7.56 \\
D-allo-Ile & 7.20 & \\
L-allo-Ile & 7.39 & \\
D -Phe & 7.31 & 7.32 \\
L-Phe & 7.19 & \\
D-Val & 7.41 & \\
L-Val & 6.98 & 7.03
\end{tabular}

